\title{
Factor analysis in optimizing the structure of the sports industry
}

\author{
Chao Chen ${ }^{1,}$ a , Bing Zhang, b \\ ${ }^{1}$ Institute of Physical Education, Jilin Normal University, Siping 136000, China \\ ${ }^{2}$ Institute of Physical Education, Huanggang Normal University, Huangzhou 438000, China
}

\begin{abstract}
Based on the statistics of the national sports in total, through the research methods of statistics from 2006 to 2008 sports and related industry data analysis, using SPSS statistical index analysis of the data to determine the effects of sports and related industry associations between, factor method was applied to further determine the relationship of index and physical output, the regression equation of the sports value are obtained.
\end{abstract}

\section{Introduction}

In order to grasp Chinese sports and relative industrial information, general administration of sport of China makes statistics of sports and relative industries in 2006-2008, and publishes statistical data. Sports industrial development is crucial to economic development, understand sports and relative industrial information has important significances in speeding up and optimizing industrial structure. Since statistical data is published, scholars' studies on sports and relative industrials have made rapidly progress [1]. Yang Qian researched sports industry and economic relations by grey relative analysis method, she pointed out that sports industry sub-factor fitness entertainment had highest correlation degree with economy, and national economy played strong leading role in sports industry development [2, 3]. Yang Qian made analytic comparison of cohabitation data, made quantitative analysis of sports and relative industrial efficiency, which provided quantization evidence for structure optimization. Liu Han-Sheng researched correlation industries internal connections by grey mathematical theory [4]. The paper based on fully referencing previous research results, adopts statistics to analyze relative industrial correlations and utilizes multiple linear methods to research on sports and relative industries.

\section{Sports and relative industries statistical data}

According to general administration of sport of China investigation, sports and relative industrial investigation have data (extract from general administration of sport of China website). During 2006 to 2008, sports and relative industrial total output value's value added has been increasing in successive years, number of employees are also continuously increasing in three years. Sports industrial rapidly development promotes civil employment, sports and relative industrial structure scale are rapidly growing [5]. Relative industrial development is wholly $\mathrm{I}$ the rising trend. But relative industrial rising amplitude is not the same, by Figure 1; it is clear about sports relative industries output value contribution rate in sports. By Table 1, it is clear about changing rate of relative industry output value, employee contribution rates in total output value and total employees during 2006 to 2008 .

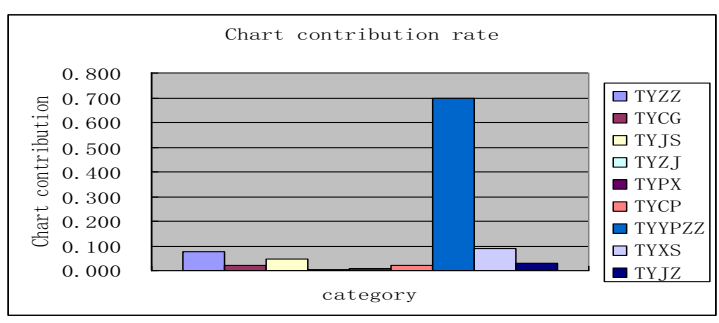

Figure 1. 2008 relative industries contribution rate

In Figure 1, TYZZ, TYCG, TYJS, TYZJ, TYPX, TYCP, TYYPZZ, TYXS, TYJZ respectively represents sports organizational management activity, stadiums management activity, sports fitness leisure activity, sports intermediary activity, sports training activity, sports lottery, sports products, clothes, shoes and caps manufacturing, sports products, clothes, shoes and caps. selling, and stadiums building. It is clear that sports lottery, sports products, clothes, shoes and caps manufacturing occupies maximum contribution rate in sports output value that occupies $70 \%$.Other industries contribution rates are less than $1 \%$. It shows sports industry structure is not reasonable.

\footnotetext{
a, b Corresponding author: ${ }^{a} 398704001 @ q q . c o m ;$ b tiyuxi@qq.com
} 
Table 1. Relative industries output values, employees' contribution rates.

\begin{tabular}{|c|c|c|c|c|c|c|}
\hline \multirow{4}{*}{ Type } & \multicolumn{6}{|c|}{ Contribution rate } \\
\hline & \multicolumn{2}{|c|}{ Year 2006} & \multicolumn{2}{|c|}{ Year 2007} & \multicolumn{2}{|c|}{ Year 2008} \\
\hline & Value added & Employee & Value added & Employee & Value added & Employee \\
\hline & $\begin{array}{l}\text { (one hundred } \\
\text { million Yuan) }\end{array}$ & $\begin{array}{l}\text { (ten thousand } \\
\text { people) }\end{array}$ & $\begin{array}{l}\text { (one hundred } \\
\text { million Yuan) }\end{array}$ & $\begin{array}{l}\text { (ten thousand } \\
\text { people) }\end{array}$ & $\begin{array}{l}\text { (one hundred } \\
\text { million Yuan) }\end{array}$ & $\begin{array}{l}\text { (ten thousand } \\
\text { people) }\end{array}$ \\
\hline $\begin{array}{c}\text { Sports organizational } \\
\text { management activity }\end{array}$ & 0.076 & 0.073 & 0.071 & 0.067 & 0.076 & 0.066 \\
\hline $\begin{array}{c}\text { Stadiums } \\
\text { management activity }\end{array}$ & 0.019 & 0.010 & 0.018 & 0.008 & 0.019 & 0.008 \\
\hline $\begin{array}{c}\text { Sports fitness leisure } \\
\text { activity }\end{array}$ & 0.048 & 0.046 & 0.046 & 0.047 & 0.048 & 0.047 \\
\hline $\begin{array}{l}\text { Sports intermediary } \\
\text { activity }\end{array}$ & 0.002 & 0.003 & 0.002 & 0.003 & 0.003 & 0.004 \\
\hline $\begin{array}{l}\text { Sports training } \\
\text { activity }\end{array}$ & 0.005 & 0.007 & 0.006 & 0.008 & 0.009 & 0.011 \\
\hline Sports lottery & 0.022 & 0.043 & 0.023 & 0.047 & 0.023 & 0.056 \\
\hline $\begin{array}{c}\text { Sports products, } \\
\text { clothes, shoes and caps } \\
\text { manufacturing }\end{array}$ & 0.717 & 0.763 & 0.710 & 0.754 & 0.700 & 0.738 \\
\hline $\begin{array}{c}\text { Sports products, } \\
\text { clothes, shoes and caps } \\
\text { selling }\end{array}$ & 0.078 & 0.043 & 0.088 & 0.054 & 0.091 & 0.058 \\
\hline Stadiums building & 0.034 & 0.011 & 0.035 & 0.012 & 0.032 & 0.011 \\
\hline
\end{tabular}

Table 2. Correlation coefficient.

\begin{tabular}{|c|c|c|c|c|c|c|c|c|c|c|}
\hline \multicolumn{11}{|c|}{ Correlation } \\
\hline & & TYZZ & TYCG & TYJS & TYZJ & TYPX & TYCP & TYYPZZ & TYXS & TYJZ \\
\hline \multirow{3}{*}{ TYZZ } & Pearson correlation & 1 & $.997^{*}$ & .995 & $.998^{*}$ & $.999^{*}$ & .959 & .983 & .978 & .919 \\
\hline & Significance(bilateral) & & .049 & .064 & .044 & .030 & .183 & .119 & .135 & .258 \\
\hline & $\mathrm{N}$ & 3 & 3 & 3 & 3 & 3 & 3 & 3 & 3 & 3 \\
\hline \multirow{3}{*}{ TYCG } & Pearson correlation & $.997^{*}$ & 1 & $1.000^{*}$ & $1.000^{* *}$ & $1.000^{*}$ & .978 & .994 & .991 & .946 \\
\hline & Significance(bilateral) & .049 & & .015 & .005 & .019 & .134 & .070 & .086 & .210 \\
\hline & $\mathrm{N}$ & 3 & 3 & 3 & 3 & 3 & 3 & 3 & 3 & 3 \\
\hline \multirow{3}{*}{ TYJS } & Pearson correlation & .995 & $1.000^{*}$ & 1 & $.999^{*}$ & $.999^{*}$ & .983 & .996 & .994 & .954 \\
\hline & Significance(bilateral) & .064 & .015 & & .020 & .034 & .119 & .055 & .070 & .194 \\
\hline & $\mathrm{N}$ & 3 & 3 & 3 & 3 & 3 & 3 & 3 & 3 & 3 \\
\hline \multirow{3}{*}{ TYZJ } & Pearson correlation & $.998^{*}$ & $1.000^{* *}$ & $.999^{*}$ & 1 & $1.000^{*}$ & .976 & .993 & .990 & .944 \\
\hline & Significance(bilateral) & .044 & .005 & .020 & & .014 & .139 & .075 & .091 & .214 \\
\hline & $\mathrm{N}$ & 3 & 3 & 3 & 3 & 3 & 3 & 3 & 3 & 3 \\
\hline \multirow{3}{*}{ TYPX } & Pearson correlation & $.999^{*}$ & $1.000^{*}$ & $.999^{*}$ & $1.000^{*}$ & 1 & .971 & .990 & .987 & .936 \\
\hline & Significance(bilateral) & .030 & .019 & .034 & .014 & & .153 & .089 & .104 & .228 \\
\hline & $\mathrm{N}$ & 3 & 3 & 3 & 3 & 3 & 3 & 3 & 3 & 3 \\
\hline \multirow{3}{*}{ TYCP } & Pearson correlation & .959 & .978 & .983 & .976 & .971 & 1 & .995 & $.997^{*}$ & .993 \\
\hline & Significance(bilateral) & .183 & .134 & .119 & .139 & .153 & & .064 & .048 & .076 \\
\hline & $\mathrm{N}$ & 3 & 3 & 3 & 3 & 3 & 3 & 3 & 3 & 3 \\
\hline \multirow{3}{*}{ TYYPZZ } & Pearson correlation & .983 & .994 & .996 & .993 & .990 & .995 & 1 & $1.000^{*}$ & .976 \\
\hline & Significance(bilateral) & .119 & .070 & .055 & .075 & .089 & .064 & & .016 & .140 \\
\hline & $\mathrm{N}$ & 3 & 3 & 3 & 3 & 3 & 3 & 3 & 3 & 3 \\
\hline \multirow{3}{*}{ TYXS } & Pearson correlation & .978 & .991 & .994 & .990 & .987 & $.997^{*}$ & $1.000^{*}$ & 1 & .981 \\
\hline & Significance(bilateral) & .135 & .086 & .070 & .091 & .104 & .048 & .016 & & .124 \\
\hline & $\mathrm{N}$ & 3 & 3 & 3 & 3 & 3 & 3 & 3 & 3 & 3 \\
\hline \multirow{3}{*}{ TYJZ } & Pearson correlation & .919 & .946 & .954 & .944 & .936 & .993 & .976 & .981 & 1 \\
\hline & Significance(bilateral) & .258 & .210 & .194 & .214 & .228 & .076 & .140 & .124 & \\
\hline & $\mathrm{N}$ & 3 & 3 & 3 & 3 & 3 & 3 & 3 & 3 & 3 \\
\hline \multicolumn{11}{|c|}{ *. It is significant correlated in 0.05 horizontal (bilateral). } \\
\hline
\end{tabular}




\begin{tabular}{|c|c|c|c|c|c|c|c|c|c|c|}
\hline \multicolumn{11}{|c|}{ Correlation } \\
\hline & & TYZZ & TYCG & TYJS & TYZJ & TYPX & TYCP & TYYPZZ & TYXS & TYJZ \\
\hline \multirow{3}{*}{ TYZZ } & Pearson correlation & 1 & $.997^{*}$ & .995 & $.998^{*}$ & $.999^{*}$ & .959 & .983 & .978 & .919 \\
\hline & Significance(bilateral) & & .049 & .064 & .044 & .030 & .183 & .119 & .135 & .258 \\
\hline & $\mathrm{N}$ & 3 & 3 & 3 & 3 & 3 & 3 & 3 & 3 & 3 \\
\hline \multirow{3}{*}{ TYCG } & Pearson correlation & $.997^{*}$ & 1 & $1.000^{*}$ & $1.000^{* *}$ & $1.000^{*}$ & .978 & .994 & .991 & .946 \\
\hline & Significance(bilateral) & .049 & & .015 & .005 & .019 & .134 & .070 & .086 & .210 \\
\hline & $\mathrm{N}$ & 3 & 3 & 3 & 3 & 3 & 3 & 3 & 3 & 3 \\
\hline \multirow{3}{*}{ TYJS } & Pearson correlation & .995 & $1.000^{*}$ & 1 & $.999^{*}$ & $.999^{*}$ & .983 & .996 & .994 & .954 \\
\hline & Significance(bilateral) & .064 & .015 & & .020 & .034 & .119 & .055 & .070 & .194 \\
\hline & $\mathrm{N}$ & 3 & 3 & 3 & 3 & 3 & 3 & 3 & 3 & 3 \\
\hline \multirow{3}{*}{ TYZJ } & Pearson correlation & $.998^{*}$ & $1.000^{* *}$ & $.999^{*}$ & 1 & $1.000^{*}$ & .976 & .993 & .990 & .944 \\
\hline & Significance(bilateral) & .044 & .005 & .020 & & .014 & .139 & .075 & .091 & .214 \\
\hline & $\mathrm{N}$ & 3 & 3 & 3 & 3 & 3 & 3 & 3 & 3 & 3 \\
\hline \multirow{3}{*}{ TYPX } & Pearson correlation & $.999^{*}$ & $1.000^{*}$ & $.999^{*}$ & $1.000^{*}$ & 1 & .971 & .990 & .987 & .936 \\
\hline & Significance(bilateral) & .030 & .019 & .034 & .014 & & .153 & .089 & .104 & .228 \\
\hline & $\mathrm{N}$ & 3 & 3 & 3 & 3 & 3 & 3 & 3 & 3 & 3 \\
\hline \multirow{3}{*}{ TYCP } & Pearson correlation & .959 & .978 & .983 & .976 & .971 & 1 & .995 & $.997^{*}$ & .993 \\
\hline & Significance(bilateral) & .183 & .134 & .119 & .139 & .153 & & .064 & .048 & .076 \\
\hline & $\mathrm{N}$ & 3 & 3 & 3 & 3 & 3 & 3 & 3 & 3 & 3 \\
\hline \multirow{3}{*}{ TYYPZZ } & Pearson correlation & .983 & .994 & .996 & .993 & .990 & .995 & 1 & $1.000^{*}$ & .976 \\
\hline & Significance(bilateral) & .119 & .070 & .055 & .075 & .089 & .064 & & .016 & .140 \\
\hline & $\mathrm{N}$ & 3 & 3 & 3 & 3 & 3 & 3 & 3 & 3 & 3 \\
\hline \multirow{3}{*}{ TYXS } & Pearson correlation & .978 & .991 & .994 & .990 & .987 & $.997^{*}$ & $1.000^{*}$ & 1 & .981 \\
\hline & Significance(bilateral) & .135 & .086 & .070 & .091 & .104 & .048 & .016 & & .124 \\
\hline & $\mathrm{N}$ & 3 & 3 & 3 & 3 & 3 & 3 & 3 & 3 & 3 \\
\hline \multirow{3}{*}{ TYJZ } & Pearson correlation & .919 & .946 & .954 & .944 & .936 & .993 & .976 & .981 & 1 \\
\hline & Significance(bilateral) & .258 & .210 & .194 & .214 & .228 & .076 & .140 & .124 & \\
\hline & $\mathrm{N}$ & 3 & 3 & 3 & 3 & 3 & 3 & 3 & 3 & 3 \\
\hline \multicolumn{11}{|c|}{ *. It is significant correlated in 0.05 horizontal (bilateral). } \\
\hline & & $* * . \mathrm{I}$ & gnifical & correlate & n .01 ho & ntal (bi & ral). & & & \\
\hline
\end{tabular}

Table 1 data shows if contribution rate is in the steady state, then the industry development level synchronizes with sports industrial development overall level, if contribution rate is in the rising states, then the industrial development level is ahead of sports industry development overall level, if contribution rate is in the diminishing state, then the industry development level falls behind sports industry development overall level. From table, it is clear that correlation industries advance developed ones that are also rapidly developing industries, there are sports products, clothes, shoes and caps selling and sports training activity. Industries that development is relative backward are sports products, clothes, shoes and caps manufacturing and stadiums building. Other sports industries developments nearly synchronize with overall development level. Industrial structure is adjusting proportions in sports total output value, manufacturing industries and building industry cools down, and other industries appear rising trend, it is clear that sports correlation industrial structure is adjusting.

\section{Sports relative industries multiple linear analysis}

\subsection{Relative industries correlation analysis}

Judge each variable correlation indicator, use correlation coefficient to express, assume there are two variables that are respectively $x_{1}, x_{2}$, according to variables data, calculate two variables correlation coefficient $r$.

Correlation coefficient absolute value is less than or equal to 1 , correlation coefficient has positive value and negative value that respectively corresponds to positive correlation and negative correlation. Operate sports relative industries statistical data in SPSS [6], and get correlation coefficient matrix Table 2In sports relative industries, correlated three other industries are sports intermediary activity, stadiums management activity and sports training activity, which shows these sports industries are closely related to sports industries totality, give priority to develop the three sports industries can promote and drive other sports industrial development. 


\subsection{SPSS correlation calculation}

In complex problems, variables numbers are quite a lot, it brings inconvenience to problem solution. Factor analysis is a method that defines fewer variables through analyzing each variable correlation. By defining common factors from variables, refine some principal components, reduce variables dimensions, and then can use fewer variable to replace all variables. Running results in SPSS is as following Table 3 .

In Table 3, result shows that first principal component feature root is 8.865 , variance contribution rate is $98.5 \%$.According to factor extracting conditions, in the analysis, principal component is one piece. Select the first factor.

Table 3. Principal component table.

\begin{tabular}{|c|c|c|c|c|c|c|}
\hline \multirow{2}{*}{$\begin{array}{c}\text { Compo } \\
\text { nent }\end{array}$} & \multicolumn{3}{|c|}{ Initial feature value } & \multicolumn{3}{|c|}{$\begin{array}{c}\text { Extract square sum and } \\
\text { input }\end{array}$} \\
\hline & Total & $\begin{array}{l}\text { Varian } \\
\text { ce } \%\end{array}$ & $\begin{array}{l}\text { Accumu } \\
\text { lation \% }\end{array}$ & $\begin{array}{l}\text { To } \\
\text { tal }\end{array}$ & $\begin{array}{l}\text { Varian } \\
\text { ce } \%\end{array}$ & $\begin{array}{l}\text { Accumu } \\
\text { lation \% }\end{array}$ \\
\hline 1 & 8.865 & 98.500 & 98.500 & $\begin{array}{c}8.86 \\
5\end{array}$ & 98.500 & 98.500 \\
\hline 2 & .135 & 1.500 & 100.000 & & & \\
\hline 3 & $\begin{array}{c}2.919 \mathrm{E}- \\
16\end{array}$ & $\begin{array}{c}3.243 \mathrm{E}- \\
15\end{array}$ & 100.000 & & & \\
\hline 4 & $\begin{array}{c}2.207 \mathrm{E}- \\
16\end{array}$ & \begin{tabular}{|c|}
$2.452 \mathrm{E}-$ \\
15
\end{tabular} & 100.000 & & & \\
\hline 5 & $\begin{array}{c}1.355 \mathrm{E}- \\
16\end{array}$ & $\begin{array}{c}1.506 \mathrm{E}- \\
15\end{array}$ & 100.000 & & & \\
\hline 6 & \begin{tabular}{|c}
$3.674 \mathrm{E}-$ \\
17
\end{tabular} & $\begin{array}{c}4.082 \mathrm{E}- \\
16\end{array}$ & 100.000 & & & \\
\hline 7 & $\begin{array}{c}-1.170 \mathrm{E}- \\
16\end{array}$ & $\begin{array}{c}-1.301 \mathrm{E}- \\
15\end{array}$ & 100.000 & & & \\
\hline 8 & $\begin{array}{c}-2.275 \mathrm{E}- \\
16\end{array}$ & $\begin{array}{c}-2.528 \mathrm{E}- \\
15\end{array}$ & 100.000 & & & \\
\hline 9 & $\begin{array}{c}-3.918 \mathrm{E}- \\
16\end{array}$ & $\begin{array}{c}-4.353 \mathrm{E}- \\
15\end{array}$ & 100.000 & & & \\
\hline \multicolumn{7}{|c|}{ Extract method: Principal component analysis. } \\
\hline
\end{tabular}

\section{Conclusion}

According to sports relative industries correlation analysis, it gets in sports industries; it should take sports intermediary activity, stadiums management activity and sports training activity as leading to promote sports industries development, by correlation analysis, it is known that some industries have no correlations, which don't conform to practice, it proves sports industrial structure irrationality, industries haven't shaped network, it has industries disjoints. Factor analysis method shows sports relative industries can be summarized as one principal component; it proves sports industries should have stronger correlations. So, sports industrial structure optimization development should take sports intermediary activity, stadiums management activity and sports training activity as leading, and meanwhile strengthen each industry associated relationships.

\section{References}

1. B. Liu, S. L. Hu, H. X. Xu and J. H. Gao, Chinese Journal of Health Policy, 2, 13-17(2009).

2. D. C. Zhang and M. Li, China Sport Science, 33, 3-23(2013).

3. Y. He and M. Xu, Journal of Chengdu Physical Education Institute, 33, 43-45(2007).

4. J. Zhang and Y. Wu, Journal of Shanghai Physical Education Institute, 6, 80-82(2012).

5. Z. L. Liu, Advances in Information Sciences and Service Sciences, 4, 210-218(2012).

6. S. S. Park, Y. T. Rhim and M. J. Kim et al, Journal of Coastal Research, 72, 96-100(2014). 